\title{
High-yield Synthesis of Transglycosylated
}

\section{Mogrosides Improves the Flavor Profile of}

\section{Monk Fruit Extract Sweeteners}

Ana Muñoz-Labrador ${ }^{1}$, Silvana Azcarate², Rosa Lebrón-Aguilar ${ }^{3}$, Jesús E. Quintanilla-López³, Plácido Galindo-Iranzo³ , Sofia Kolida $^{4}$, Lisa Methven ${ }^{5}$, Robert A. Rastall ${ }^{5}$, F. Javier Moreno*1, Oswaldo Hernandez-Hernandez ${ }^{1}$

Madrid, Spain; ana.munoz@csic.es (A. M-L); javier.moreno@csic.es (F.J. M.);

o.hernandez@csic.es (O.H-H) Consejo Nacional de Investigaciones Científicas y Técnicas (CONICET), Godoy Cruz 2290 CABA (C1425FQB), Argentina; silvanaazcarate@gmail.com (S.A) Institute of Physical Chemistry 'Rocasolano’ (IQFR-CSIC), Serrano 119, 28006 Madrid, Spain; rlebron@iqfr.csic.es (R. L-A); je.quintanilla@iqfr.csic.es (JE. Q-L); pgalindo@iqfr.csic.es (P. G-I) OptiBiotix Health plc, Innovation Centre, Innovation Way, Heslington, York, Y010 5DG, United Kingdom; skolida@optibiotix.com (S.K) Department of Food and Nutritional Sciences, The University of Reading, PO Box 226, Whiteknights, Reading, RG6 6AP, United Kingdom; l.methven@reading.ac.uk (L. M); r.a.rastall@reading.ac.uk (R. R)

* $\quad$ Correspondence: javier.moreno@csic.es; Tel.: +34-910017948 (F.J. M.) 


\section{Supplementary figures:}

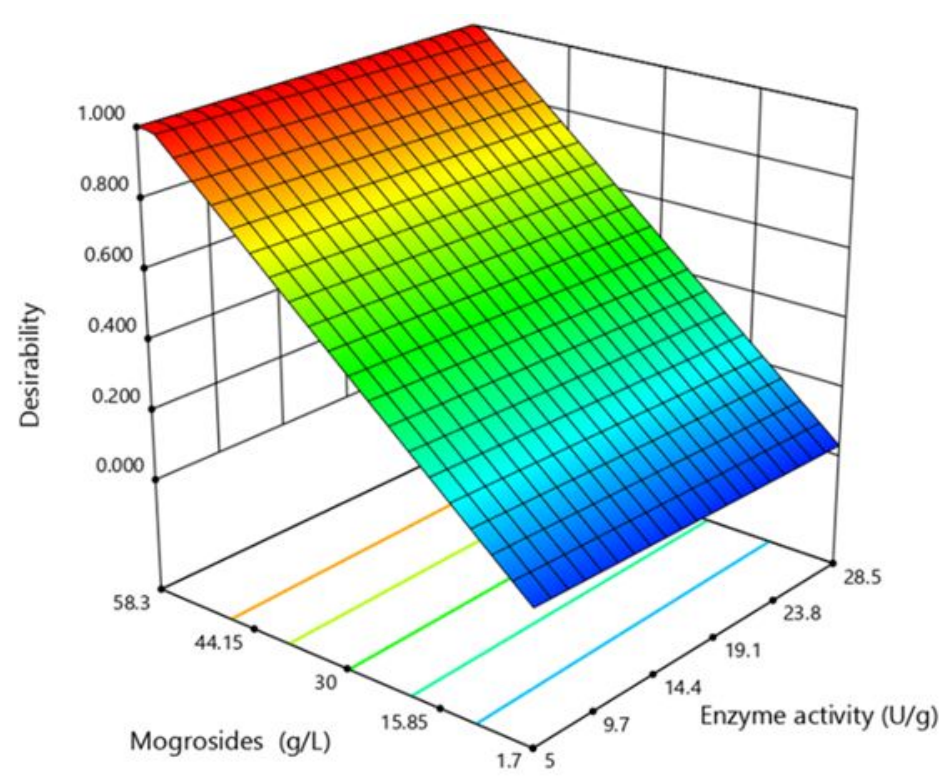

Figure S1. Three-dimensional plot showing the effect of initial unmodified mogrosides and the enzymatic activity on the desirability to maximize the concentration of synthesized glucosylated mogrosides. 

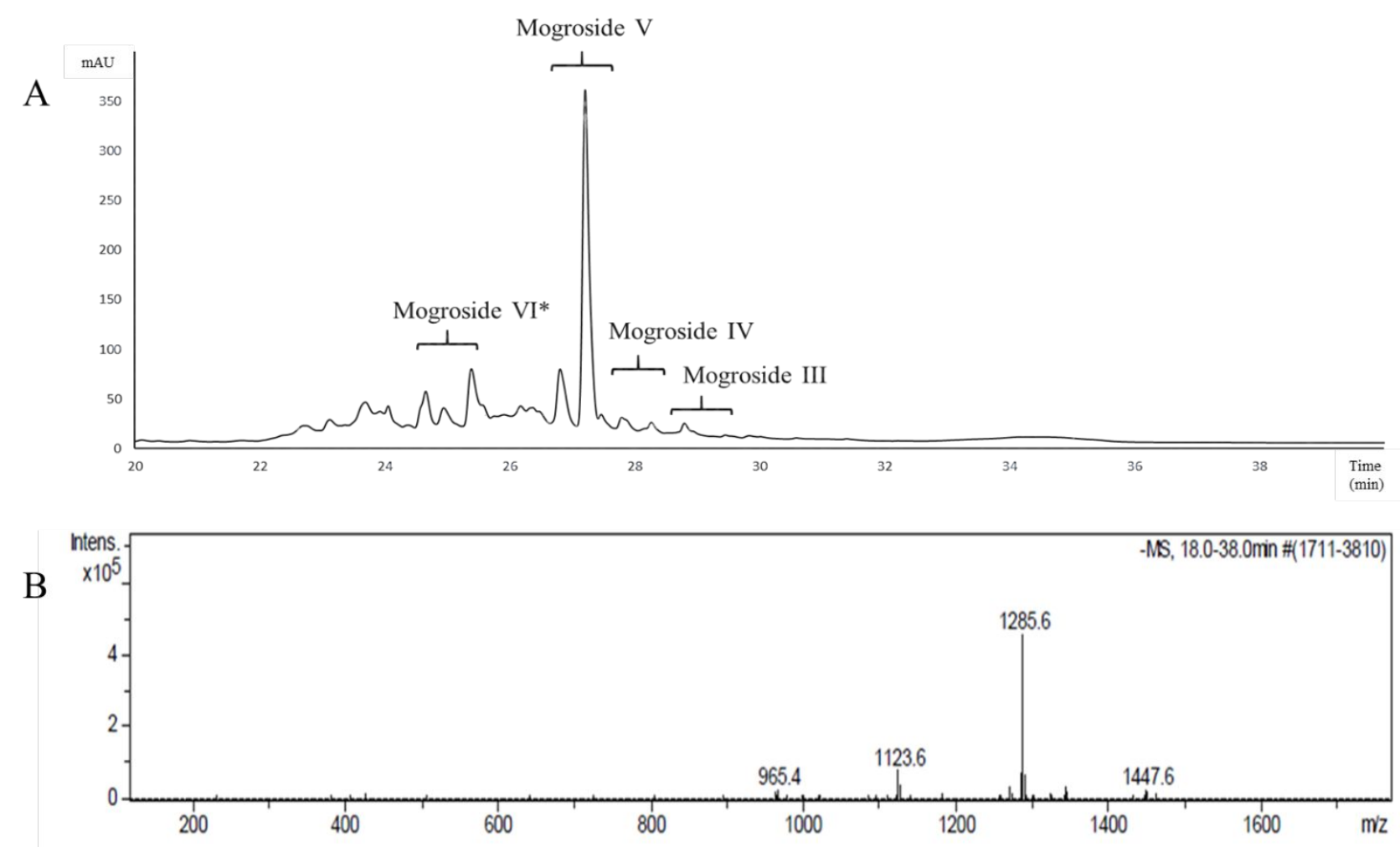

Figure S2. (A) LC-DAD chromatogram of unmodified Luo-Han-Guo fruit extract. The identified compounds correspond to Mogrosides V, VI*, IV, and III. *Other minor non-identified mogrosides. (B) LC-MS spectrum corresponding to the cucurbitane triterpene glycosides found in the unmodified Luo-Han-Guo fruit extract: 1285.6 $m / z$ - Mogroside V, $1447.6 \mathrm{~m} / z$ - Mogroside VI, $1123.6 \mathrm{~m} / z$ - Mogroside IV, and $965.4 \mathrm{~m} / \mathrm{z}$ - Mogroside III. 